\title{
Retrospektive Studiendaten favorisieren Methotrexat versus Mycophenolatmofetil
}

Fragestellung: Diese französische Studie untersuchte, ob die Gabe von Mycophenolatmofetil in der Therapie der Neurosarkoidose ebenso wirksam ist wie die Behandlung mit Methotrexat.

Hintergrund: Die Sarkoidose führt in etwa $5 \%$ der betroffenen Patienten zu einer Beteiligung des Nervensystems [1]. Es können alle Teile des ZNS als auch des peripheren Nervensystems involviert sein. Die akute Neurosarkoidose wird dabei üblicherweise so lange mit Kortikosteroiden behandelt, bis eine Remission eingetreten ist. Als kortisonsparendes Medikament wurde bisher häufig Methotrexat empfohlen. In den letzten Jahren wurden zunehmend auch andere Medikamente - vor allem Mycophenolatmofetil - eingesetzt, ohne dass es dazu randomisierte Studien gegeben hätte.

Patienten und Methodik: Es wurde eine retrospektive Multicenter-Studie zur Wirksamkeit von Methotrexat versus Mycophenolatmofetil bei Patien-

Bitoun S, Bouvry D, Borie R et al. Treatment of neurosarcoidosis: A comparative study of methotrexate and mycophenolate mofetil. Neurology 2016; 97: $2517-21$ ten mit histologisch bewiesener Neurosarkoidose durchgeführt. Insgesamt schloss die Studie 40 Patienten ein. Ausgewertet wurden $14 \mathrm{mit}$ Mycophenolatmofetil und 32 mit Methotrexat behandelte
Patienten, die eine der Therapien über mindestens drei Monate erhielten. Sechs der Patienten bekamen sequenziell beide Medikamente. Die Effektivität der Therapie wurde in den Monaten bis zum einem erneuten Schub gemessen.

Ergebnisse: Die Rate der erneuten Schübe betrug bei den mit Methotrexat behandelten Patienten $47 \%$ (0,2 Schübe/Jahr), bei den mit Mycophenolatmofetil behandelten Patienten lag die Schubrate bei $79 \%$ (0,6 Schübe pro Jahr). Die mittlere Zeit bis zum nächsten Schub war in der Methotrexat-Gruppe signifikant länger mit 28 Monaten gegenüber der MycophenolatmofetilGruppe mit elf Monaten. Die mediane Steroid-Dosis zum Zeitpunkt des erneuten Schubs lag in der MycophenolatmofetilGruppe mit $5 \mathrm{mg} /$ Tag deutlich niedriger als in der MethotrexatGruppe mit 12,5 mg/Tag.

Bezüglich der Nebenwirkungen gab es keine statistisch signifikanten Unterschiede. Es mussten jedoch in der MethotrexatGruppe zwei Patienten aufgrund schwerer Infektionen das Medikament absetzen, dagegen keiner in der Mycophenolatmofetil-Gruppe.

Schlussfolgerungen: Die Autoren folgern, dass Methotrexat gegenüber Mycophenolatmofetil präferenziell zur Remissionserhaltung bei Patienten mit Neurosarkoidose eingesetzt werden sollte und die Studie eine Klasse-IV-Evidenz für diese Schlussfolgerung gibt.

\section{Nur eine randomisierte klinische Studie kann diese Fragestellung klären}

Es ist erstaunlich, dass diese Arbeit trotz der methodischen Mängel in Neurology publiziert wurde. Die Studie hatte einen retrospektiven Ansatz, die Anzahl der Patienten war sehr niedrig, die beiden Studiengruppen waren äußerst heterogen bezüglich der Art der Neurosarkoidose (50\% der Patienten in der Mycophenolatmofetil-Gruppe hatten eine ZNS-Beteiligung, bei nur 35\% war das in der Methotrexat-Gruppe der Fall, dagegen waren die Hirnnerven bei $46 \%$ der Patienten in der Methotrexat-Gruppe betroffen gegenüber $25 \%$ in der Mycophenolatmofetil-Gruppe) und hinsichtlich der begleitenden Steroid-Dosis gab es erhebliche Unterschiede. Insofern kann aus der Studie nur geschlossen werden, dass beide Medikamente eingesetzt werden können und die Verträglichkeit von Mycophenolatmofetil besser zu sein scheint als die von Methotrexat. Um die Fragestellung der Studie zu klären, würde es einer randomisierten klinischen Studie bedürfen, die vor allem auch die Art der Neurosarkoidose und die Steroid-Dosis sowie die Art der Vorbehandlung zwischen den Gruppen matcht.
In den DGN-Leitlinien werden weiterhin Methotrexat und Azathioprin empfohlen, wobei sich die Auswahl des Medikaments nach den Komorbiditäten und dem früheren Ansprechen auf Therapien richten sollte [2].

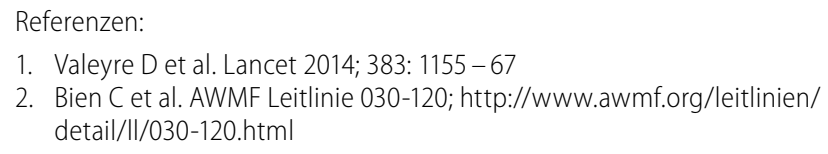
detail/ll/030-120.html

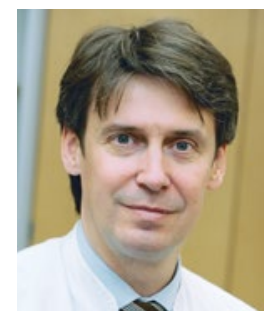

Prof. Dr. med. Matthias Maschke, Trier

Chefarzt der Abteilung für Neurologie und Neurophysiologie, Krankenhaus der Barmherzigen Brüder Trier E-Mail:m.maschke@bk-trier.de 